\title{
openheart Nurse-coordinated care improves the achievement of LDL cholesterol targets through more intensive medication titration
}

\author{
Marjolein Snaterse, ${ }^{1}$ Harald T Jorstad, ${ }^{2}$ Marlies Heiligenberg, ${ }^{1}$ Gerben ter Riet, ${ }^{3}$ \\ S Matthijs Boekholdt, ${ }^{2}$ Wilma Scholte op Reimer, ${ }^{1,2}$ Ron J Peters ${ }^{2}$
}

To cite: Snaterse M, Jorstad HT, Heiligenberg $\mathrm{M}$, et al. Nursecoordinated care improves the achievement of LDL cholesterol targets through more intensive medication titration. Open Heart 2017;4:e000607. doi:10.1136/ openhrt-2017-000607

Received 3 February 2017 Revised 17 May 2017 Accepted 13 June 2017

\section{CrossMark}

${ }^{1}$ ACHIEVE Centre for Applied Research, Faculty of Health, Amsterdam University of Applied Sciences, Amsterdam, Netherlands

2Department of Cardiology, Academic Medical Center, University of Amsterdam, Amsterdam, Netherlands ${ }^{3}$ Department of General Practice Academic Medical Center, Amsterdam, Netherlands

Correspondence to Marjolein Snaterse; $\mathrm{m}$. snaterse@hva.nl,m.snaterse@ amc.uva.nl

\section{ABSTRACT}

Background Nurse-coordinated care (NCC) improves the achievement of low-density lipoprotein-cholesterol (LDL-C) targets after an acute coronary syndrome (ACS). We hypothesised that NCC improves achievement of LDL-C targets through more intensive medication titration. Methods We used data from Randomised Evaluation of Secondary Prevention by Outpatient Nurse Specialists (RESPONSE), a multicentre randomised trial on the efficacy of NCC in 754 ACS patients. Follow-up data were collected at 6 and 12 months. To enable comparison between the various types and dosages of statins, we used the average lipid-lowering potency (ALLP, \% LDL-C lowering) as an indicator of lipid-lowering medication intensity.

Results Most patients in NCC intervention and usual care groups (96\%) had started lipid-lowering therapy during the index hospitalisation. At 6 months, titration activities (up or down) were applied in $45 \%$ of NCC patients compared with $24 \%$ of patients receiving usual care $(p<0.001)$, and a difference was also seen at 12 months follow-up (52\% vs $34 \%, p<0.001)$. In patients not on LDL-C target at baseline, titration activities at 6 months were recorded in $63 \%$ and $30 \%$ of NCC and usual care patients respectively $(p<0.001)$, with increased titration activities in both groups at 12 months $(69 \%$ vs $43 \%, p<0.001)$.

Conclusion NCC is associated with more frequent and intense lipid-lowering medication titration to reach LDL-C targets as compared with usual care alone. Further, merely starting the guideline-recommended dose is insufficient to reach the guideline-recommended LDL-C target level. Trial Registration number: TC1290 (Netherlands).

\section{INTRODUCTION}

Among patients with coronary heart disease (CHD), treatment of risk factors is the cornerstone of secondary prevention. ${ }^{1}$ In the last decade, a substantial increase in antihypertensive and lipid-lowering medication prescriptions has been observed. ${ }^{2}$ Despite a substantial increase in the number of patients receiving guideline-recommended medication, the European Action on Secondary and Primary Prevention by Intervention to Reduce Events (EUROASPIRE) survey

\section{KEY QUESTIONS}

What is already known about this subject? Nurse-coordinated care improves the achievement of low-density lipoprotein-cholesterol (LDL-C) targets after an acute coronary syndrome (ACS)

What does this study add?

Nurse-coordinated care is associated with more frequent and intense lipid-lowering medication titration to reach LDL-C targets as compared with usual care alone.

How might this impact clinical practice? Merely starting the guideline-recommended dose is insufficient to reach the guideline-recommended LDL-C target level. Nurse-coordinated care, combined with guideline-based titration recommendations, improves ACS patient outcomes.

showed that up to 3 years after hospitalisation, two-thirds of patients have uncontrolled hypertension, and only half of the patients achieve the guideline-recommended target level for low-density lipoprotein-cholesterol (LDL-C). ${ }^{3}{ }^{4}$ It has been hypothesised that factors contributing to this suboptimal risk factor control include prescriptions with inadequate dosage, inadequate up-titration of medication, poor adherence of patients to recommended lifestyle changes, poor medication compliance and low standards of follow-up care. ${ }^{5}$

Nurse-coordinated care (NCC) has shown to be a promising strategy to improve secondary prevention, and is currently recommended in the 2016 European prevention guidelines. ${ }^{1}$ In line with this recommendation, we found in a recent systematic review that NCC programmes successfully reduce systolic blood pressure and LDL-C. ${ }^{6}$ However, a clear understanding of how NCC improves achievement of LDL-C targets is still needed. More specifically, no studies have investigated 
the effect of medication titration in NCC, but it has been hypothesised that medication titration could cause this effect. ${ }^{7}$

To address this gap in knowledge, we investigated the process of medication titration in the treatment of LDL-C in NCC. We used data from the Randomised Evaluation of Secondary Prevention by Outpatient Nurse Specialists (RESPONSE) trial (see below). As the lifestyle risk factors were comparable in both groups in the study, the previously reported improvement of the proportion of patients on target for LDL-C in the NCC intervention group could not be explained by lifestyle changes. Additionally, participating nurses in this trial reported that the NCC intervention allowed them more frequent contact with patients and the opportunity to monitor targets more carefully. ${ }^{8}$ We therefore hypothesised that lipid-lowering medication titration activities occurred more often in the NCC than usual care group, and that this led to better achievement of LDL-C targets.

\section{METHODS}

\section{Study design and population}

We used data from the RESPONSE trial, a multicentre randomised clinical trial including 754 patients from 11 centres in the Netherlands. ${ }^{9}$ The study was designed to quantify the impact of a practical, hospital-based nurse-coordinated prevention programme on cardiovascular risk in patients discharged after an acute coronary syndrome (ACS), as compared with usual care alone. Patients aged 18-80 years were eligible if they had been diagnosed with ACS within 8 weeks prior to entry into the trial. Patients were excluded if they (1) were unable to visit the nurse-coordinated prevention programme, (2) were not available for follow-up, (3) had a limited life expectancy ( $\leq 2$ years), and (4) were diagnosed with heart failure New York Heart Association class III or class IV.

\section{Nurse-coordinated care}

Nurses participating in the NCC programme were registered nurses with at least a 4 years bachelor's degree in nursing. They had experience in cardiovascular care and were trained in motivational interviewing. Patients in the NCC group visited the outpatient clinic up to four times during the first 6 months after inclusion, in addition to outpatient clinic visits to their cardiologist (usual care). During each nurse visit, cardiovascular risk factors were assessed, lipid profiles (including LDL-C) were reviewed, medication therapy evaluated and patient compliance with medical treatment and lifestyle recommendations was encouraged. To achieve the target lipid levels, the nurses were also encouraged to titrate medication in collaboration with the treating cardiologist.

\section{Data collection}

Data on clinical and demographic characteristics and CHD risk factors were collected at baseline and at 6 and 12 months after randomisation. Baseline measurements were performed within 8 weeks after ACS. Patients were enrolled at an average of 4 weeks (SD 2.7) after the ACS. Data on medication use was collected at baseline, 6 months and 12 months follow-up. The data on lipid-lowering medication included number of lipid-lowering medications and, for each medication, the generic name, dosage and frequency. When LDL-C was not on target during the four NCC visits, nurses documented when medication was changed during the NCC visit, and if the treating specialists were consulted and/or patients were referred to treating specialists. All venous blood measurements were taken after a minimum of 8 hours of fasting. The target for LDL-C level was $\leq 2.5 \mathrm{mmol} / \mathrm{L}$, as recommended by the national CVD prevention guideline at that time. ${ }^{10}$ Dyslipidaemia was defined by the following criteria: a history of deviated serum cholesterol values (LDL-C $>4 \mathrm{mmol} / \mathrm{L}$, HDL-cholesterol $<1.0 \mathrm{mmol} / \mathrm{L}$, triglycerides $>2 \mathrm{mmol} / \mathrm{L}$ or total cholesterol $>5 \mathrm{mmol} / \mathrm{L}$ ) or treatment for dyslipidaemia. Further details on the trial have been published previously. ${ }^{911}$

\section{Lipid-lowering medication intensity and titration}

Our main outcome of interest was the proportion of patients with up-titration or down-titration activities in the NCC compared with usual care, assessed by changes in lipid-lowering medication intensity at 6 months and 12 months, relative to baseline medication intensity. The 6 months follow-up visit was performed directly after completion of the NCC intervention (ie, after up to four NCG visits), while between 6 and 12 months follow-up, no specific interventions took place in either group. To account for the use of different lipid-lowering agents and dosages, the intensity of each prescription was expressed as a potential average lipid-lowering potency (ALLP, \% LDL-C lowering) ranging from 13 to $70 .{ }^{12}$ ALLP and up-titration or down-titration was measured at 6 and 12 months follow-up. Up-titration was defined as an increase in ALLP as compared with baseline ALLP, whereas down-titration was defined as a decrease in ALLP.

As the Dutch guideline for cardiovascular risk management recommends starting with simvastatin $40 \mathrm{mg}$ daily when patients are diagnosed with $\mathrm{ACS},{ }^{13}$ we defined simvastatin $40 \mathrm{mg}$ as the lowest recommended dose approved for the management of ACS.

\section{Statistical analysis}

Comparisons between groups were performed using $\chi^{2}$ test for categorical variables. Differences between characteristics of up-titrated and down-titrated patients were analysed by the $\chi^{2}$ test. The $p$ values presented in figure 1 were up-titration versus no titration (none), and down-titration versus no titration (none). A two-sided $p$ value of $<0.05$ was considered statistically significant. As ALLP is not a continuous variable, we expressed ALLP as a sum of the prescribed potencies per group. SPSS Statistics for Macintosh, V.22.0. (Armonk, New York, USA) was used for descriptive statistical analyses. 


\section{6 months follow-up}

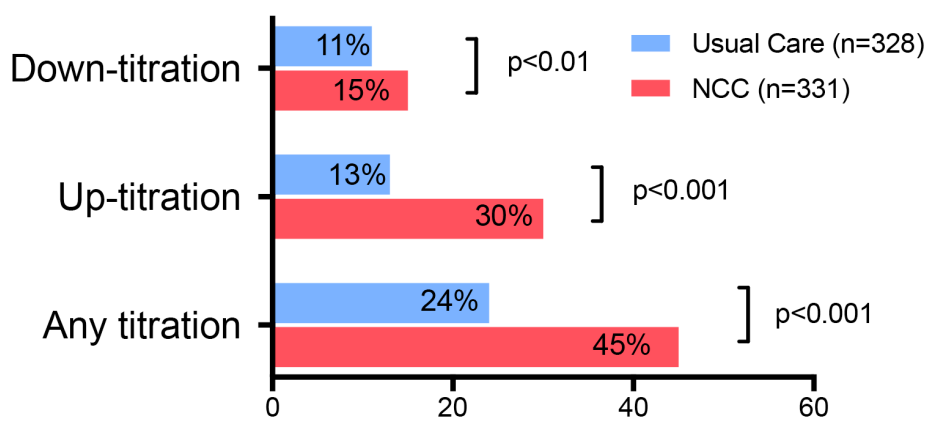

All patients

\section{2 months follow-up}

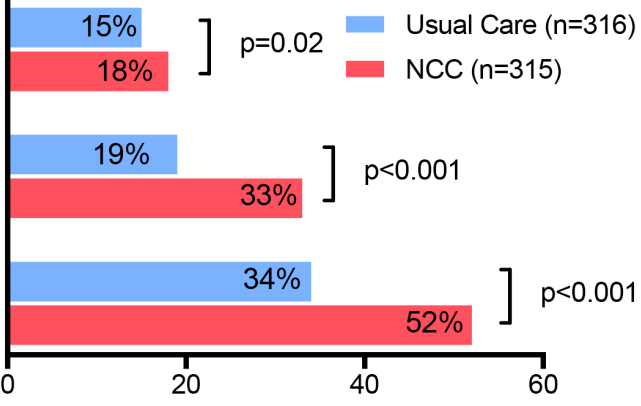

Figure 1 Titration activities from baseline up to 6 and 12 months follow-up in nurse-coordinated care (NCC) versus usual care patients. X-as: patients (percentage), Y-as: titration activities. Up-titration and down titrations are relative to baseline. Percentages are $\%$ of total population (upper panel) and $\%$ of population not on target (lower panel). All $p$ values are calculated with the relevant parameter (down-titration, up-titration or any titration) versus no titration (none). Upper panel: percentage of patients with titrations of total population. All patients: Usual care at 6 months $n=328, N C C$ at 6 months $n=331$, usual care at 12 months $n=316, N C C$ at 12 months $n=315$. Lower panel: percentage of patients with titrations of patient population not on lowdensity lipoprotein-cholesterol (LDL-C) target at baseline: Usual care at 6 months $n=94, N C C$ at 6 months $n=100$; usual care at 12 months $n=89, N C C$ at 12 months $n=90$. Not on target is defined as LDL-C $>2.5 \mathrm{mmol} / \mathrm{L}$. Analysis applied for patients on lipid-lowering medication and patients with complete medication data.

In order to include the NCG intervention effect at 6 months, we plotted ALLP changes between baseline and 6 months. We assessed if patients in the NCC group who were (not) on target at baseline received greater intensity changes than those in the usual care group by estimating the interaction between treatment arm and (not) being on target at baseline in a linear regression analysis. These analyses were performed using Stata V.13.1 (College Station, Texas, USA).

To check for selective dropout, we used a logistic regression model and regressed a binary variable indicating missingness $(1=y e s, 0=$ no $)$ on the following variables as predictors of missingness under the hypothesis that if all ORs were close to 1, selective dropout due to these predictors is unlikely: age, gender, education level, index event, history of CVD, alcohol, smoking at baseline, diabetes mellitus and their interaction with randomisation group.

\section{RESULTS}

Our population consisted of 754 patients with a mean age of 58 years (SD 10.1), $80 \%$ were men. The majority ( $73 \%$ ) had no history of CVD prior to the index hospitalisation. As previously described, baseline patient characteristics did not differ between the NCC and usual care groups. ${ }^{9}$ In the NCC group, $92 \%$ of 365 patients attended all four NCC consultations as scheduled during the first 6 months. In total, 46 patients in the intervention and 33 patients in the usual care group had one or more missing values for our analyses (11\%). Logistic regression did not reveal an indication for selective dropout between the NCC and usual care group. 


\section{Titration activity outcome}

The proportion of patients with up-titration or down-titration of lipid-lowering medication from baseline to 6 and 12 months follow-up was higher in the NCC group as compared with the usual care group (figure 1). Reflective of the NCC titration intervention, markedly more lipid-lowering titration was seen at 6 months follow-up in the NCC group compared with the usual care group (any titration in all patients $45 \%$ vs $24 \%$, $\mathrm{p}<0.001$ ) (figure 1). At 12 months, a slight increase of titration activities was seen in both groups, yet a statistically significant difference between the two groups remained (52\% vs $34 \%, \mathrm{p}<0.001)$. While both up-titration and down titration in ALLP were seen in both groups, more patients in the NCC than in the usual care group were up-titrated (6 months $30 \%$ vs $13 \%$, $\mathrm{p}<0.001 ; 12$ months $33 \%$ vs $19 \% \mathrm{p}<0.001)$.
In patients not on LDL-C targets at baseline (figure 1), most titration activities (up or down) and the largest difference between NCC and usual care groups were observed in the first 6 months (6 months: $63 \%$ vs $30 \%$, $\mathrm{p}<0.001 ; 12$ months: $69 \%$ vs $43 \%, \mathrm{p}<0.001)$. Similarly, in patients not on target at baseline, also up-titration activities were more often observed in the NCC than in the usual care group, particularly in the first 6 months (6 months: $51 \%$ vs $24 \%, \mathrm{p}<0.001 ; 12$ months: $58 \%$ vs $33 \%, \mathrm{p}<0.001)$.

figure 2 shows all ALLP changes between baseline and 6 months as a function of LDL-C at baseline for NCC and usual care patients (not) on target at baseline. On average, NCC had an (absolute) effect on ALLP compared with usual care alone, especially if patients were not on target at baseline (slope 2.3, (95\% -0.11 4.72)). The differences in SD between NCC and usual
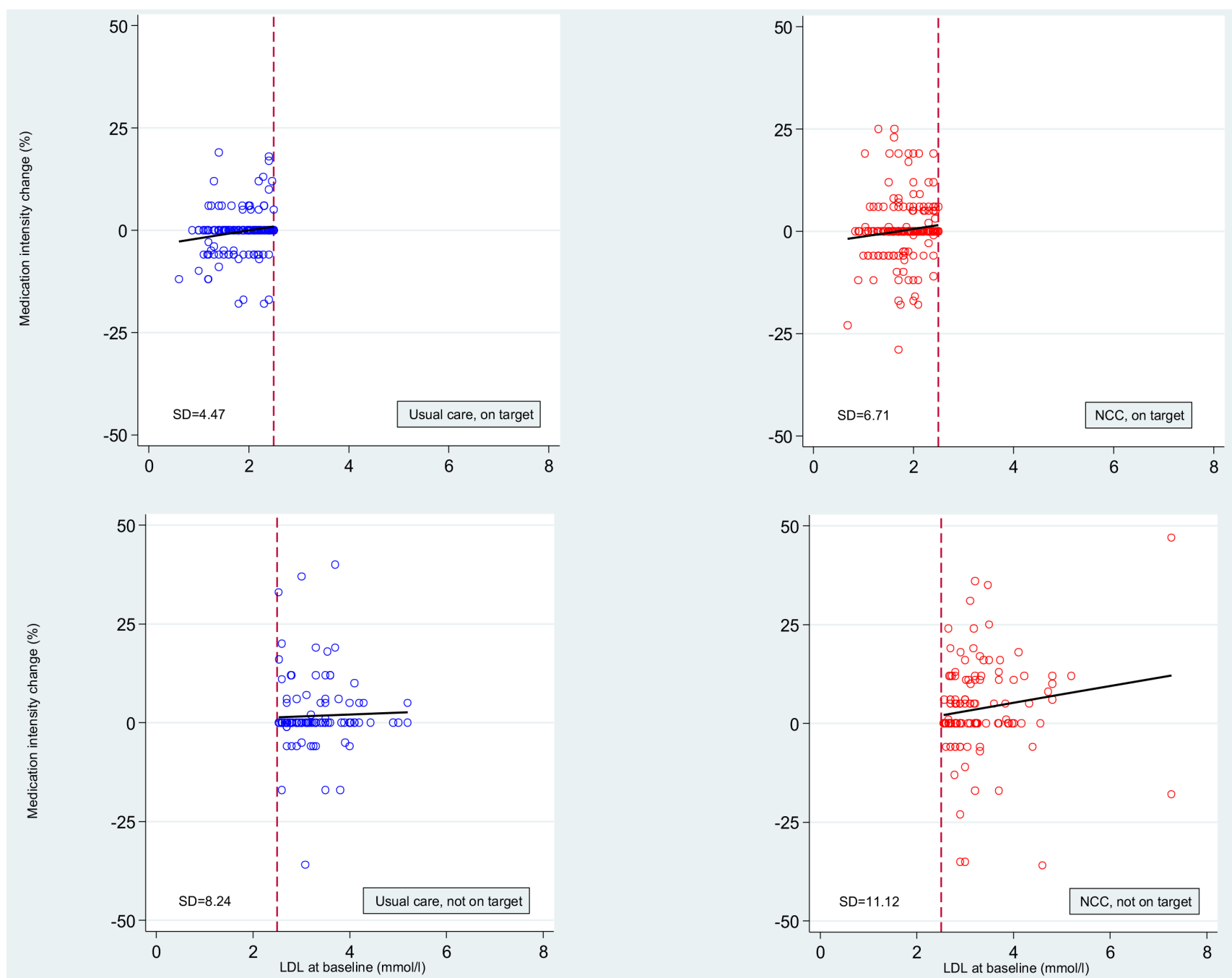

Figure 2 Medication intensity (ALLP) changes between baseline and 6 months, by (not) being low-density lipoproteincholesterol (LDL-C) target at baseline for nurse-coordinated care (NCC) (red dots) and usual care (blue dots) patients. Dots represent individual patients. The right lower graph shows, on average, more medication intensity changes in NCC patients not on target at baseline compared with usual care patients (left). The red dashed vertical lines indicate the cut-off LDL-C serum concentration of $2.5 \mathrm{mmol} / \mathrm{L}$. The black lines are the slopes based on a linear regression analysis of the medication intensity changes against LDL-C levels at baseline. ALLP, the average lipid-lowering potency (ALLP, \% LDL-C lowering) as an indicator of lipid-lowering medication intensity; LDL, low-density lipoprotein. 
Table 1 Low-density lipoprotein-cholesterol (LDL-C) and the average lipid-lowering potency (ALLP) in nurse-coordinated care (NCC) versus usual care patients at baseline and 6 and 12 months follow-up

\begin{tabular}{|c|c|c|c|c|c|c|c|c|}
\hline & Baseline* & & F6 & & & F12 & & \\
\hline Parameter & $\begin{array}{l}\text { NCC } \\
(n=365)\end{array}$ & $\begin{array}{l}\text { Usual care } \\
(n=367)\end{array}$ & $\begin{array}{l}\text { NCC } \\
(n=356)\end{array}$ & $\begin{array}{l}\text { Usual care } \\
(n=346)\end{array}$ & p Value & $\begin{array}{l}\text { NCC } \\
(n=357)\end{array}$ & $\begin{array}{l}\text { Usual care } \\
(n=352)\end{array}$ & p Value \\
\hline $\begin{array}{l}\text { On lipid- } \\
\text { lowering } \\
\text { medication, } \\
\mathrm{n}(\%)\end{array}$ & 350 (96\%) & $352(96 \%)$ & 345 (96\%) & 335 (96\%) & 0.70 & 331 (93\%) & $328(94 \%)$ & 0.64 \\
\hline $\begin{array}{l}\text { LDL-C OT } \\
(\leq 2.5 \mathrm{mmol} / \mathrm{L})\end{array}$ & 247 (68\%) & $249(68 \%)$ & $284(80 \%)$ & $241(69 \%)$ & $<0.001$ & $263(74 \%)$ & $223(64 \%)$ & $<0.01$ \\
\hline $\begin{array}{l}\text { Total ALLP } \ddagger \\
\text { (\% LDL-C } \\
\text { lowering) }\end{array}$ & 14.366 & 13.943 & 15.003 & 14.030 & NA & 14.564 & 13.964 & NA \\
\hline
\end{tabular}

${ }^{*}$ At baseline differences not statistically significant at the $5 \%$ level.

†Calculated between NCC and usual care (between-groups).

$\ddagger A L L P$ : the ALLP as an indicator of lipid-lowering medication intensity using the method by Besseling et al ${ }^{12}$ (ref).

Total ALLP is the sum of the prescribed lipid-lowering potencies (\%) per group.

NA, not applicable; OT, on target.

care reaffirm the spread of ALLP between these two groups.

\section{Lipid-lowering medication data}

At baseline, the proportion of patients on lipid-lowering medication was high in both the NCC (96\%) and the usual care group (96\%), and $68 \%$ of all patients were on LDL-C target at baseline (table 1). Simvastatin (43\%), followed by atorvastatin $(41 \%)$, were the most commonly used lipid-lowering medications prescribed at baseline. During follow-up, a higher proportion of patients in the NCC group were on target compared with the usual care group (6 months: $80 \%$ vs $69 \%, \mathrm{p}<0.001 ; 12$ months: $74 \%$ vs $64 \%, \mathrm{p}<0.01)$. Total ALLP was slightly higher in the NCC as compared with usual care at both 6 months (15.003 vs 14.030) and 12 months (14.564 vs 13.964) (table 1).

\section{Characteristics of up- titrated and down-titrated patients compared with patients with no titration}

There were no differences in demographic or clinical characteristics as age, gender, level of education, index event or cardiovascular risk factors of up-titrated and down-titrated patients (data not shown). However, up-titrated patients had dyslipidaemia more frequently as compared with patients with no titration $(79 \%$ vs $70 \%$, respectively, $\mathrm{p}=0.04$ ), and up-titrations were associated with allocation to the NCC group (62\% vs $43 \%$, $\mathrm{p}<0.001)$.

Down-titrated patients had dyslipidaemia less frequently as compared with patients with no titration $(56 \%$ vs $70 \%$, respectively, $\mathrm{p}=0.02$ ). Down-titration was also more frequently seen in patients allocated to the NCC group as compared with patients with no titration ( $55 \%$ vs $43 \%$, respectively, $\mathrm{p}=0.02$ ).

\section{DISCUSSION}

Our study demonstrates that NCC in patients with ACS is associated with more frequent lipid-lowering medication titration and with higher ALLP values to reach LDL-C targets as compared with usual care alone. These titrations took place in a relatively short amount of time (four visits in 6 months after an ACS), but changes made in the first 6 months in lipid-lowering medication were also observed 6 months after completion of the NCC programme, and were reflected in a higher proportion of patients reaching targets for LDL-C. Our study took place in a context of high prescription rates of lipid-lowering medication (96\% in both groups at baseline). Despite these high prescription rates, the target for LDL-C $(2.5 \mathrm{mmol} / \mathrm{L})$ was not reached in a considerable number of patients in both groups (NCC $26 \%$ vs usual care $36 \%$ ). Our study shows that there is considerable room for individual tailoring of lipid-lowering medication therapy, with more both up-titration and down-titration in medication intensity in the NCC group. While lifestyle modification could account for some changes in LDL-C levels, it is unlikely that this can explain the differences in the higher proportion of patients on target in the NCC group, as lifestyle risk factors were comparable through the study up until 12 months follow-up. ${ }^{9}$ Despite a small difference in the total sum of ALLP in both groups at 6 and 12 months, the proportion of individuals on target for LDL-C was markedly higher in the NCC group as compared with the usual care group, reflecting the efficacy of adequate individual medication titration.

Large proportions of high-risk cardiovascular patients have been shown to discontinue their statin therapy, emphasising the need for healthcare providers to discuss medication use with their patients. ${ }^{14}$ An integral part of the NCC intervention in our study was interviewing patients about their compliance, asking about barriers 
concerning adherence and titrating medication (ie, lipid lowering medication) to optimise adherence. Our data showed that down-titrations were made in NCC patients. A possible reason for these down-titrations could be maintaining compliance in case of side effects, as patients on high-intensity statin therapy who experience side effects (such as myopathy) are likely to be less compliant than patients down-titrated to a better tolerated statin intensity.

According to the European Society of Cardiology (ESC) guideline, reducing dosage is an effective approach for enhancing medication adherence. ${ }^{15}$ Nurse-coordinated programmes are associated with modest but positive effects on reducing cholesterol levels according to recent meta-analyses. ${ }^{6}{ }^{16}$ However, studies assessing patients' medication adherence found improved patient adherence in one study ${ }^{17}$ and no differences between NCC and usual care in two other studies. ${ }^{18} 19$ Reasons for poor patient adherence are multifactorial. According to the WHO, reasons for medication non-adherence are categorised in five groups: health system, condition, patient, therapy and socioeconomic factors. ${ }^{15}$ In particular, education and frequent follow-up visits have been shown to be associated with improved adherence, ${ }^{20}$ and NCC potentially positively influences several of these categories. While we found that targets for LDL-C were more frequently achieved in NCC, more research on the role of NCC to improve medication adherence in general would be valuable.

Patients allocated to the NCC group reached the target level of LDL-C in a short period of time after discharge. This is likely to be beneficial, as several trials have demonstrated important reductions in major cardiovascular events from lowering cholesterol, especially LDL-C. ${ }^{21}$ The total sum of ALLP for NCC patients was only slightly higher compared with patients in usual care. This should be seen as clinically relevant as this difference probably led to a larger proportion of patients achieving target level for LDL-C, and the clinical benefits of LDL-C lowering in general are well known. 223

Secondary prevention based on nurses' collaboration has the potential to improve patient care. While healthcare organisations differ widely across Europe, the ESC prevention guidelines recommend a multidisciplinary team for secondary prevention including physicians and nurses. In some countries, secondary prevention is mainly the task of physicians, while in others, specially educated and trained nurses play a more prominent role. ${ }^{1}$ Physicians and nurses are recommended to work together as a team to provide the most effective multidisciplinary care. NCC has proven to be effective in reducing risk factors, ${ }^{6}$ ${ }^{9}$ anxiety and depression, ${ }^{24}$ and nurses reported to appreciate participating in such multidisciplinary teams. ${ }^{8}$ Therefore, depending on local practice, integrating NCC should be considered in secondary prevention in ACS patients.

\section{New developments and limitations}

The ESC guideline target for LDL-C changed from $2.5 \mathrm{mmol} / \mathrm{L}$ to $1.8 \mathrm{mmol} / \mathrm{L}$ after the completion of inclusion of patients in the RESPONSE trial. ${ }^{15}$ This change increases the need for new initiatives to reach LDL-C targets in patients with CHD, as it is shown that only a minority of patients reach these stricter targets. ${ }^{4}$ The specific role of NCC in this process needs further evaluation, especially with the upcoming availability of new pharmacological strategies, such as PCSK9-inhibitors.

Moreover, it should be noted that our data on medication use were based on self-report by professionals, and not corroborated with additional questionnaires regarding adherence or pill counts. While side effects were discussed with patients during NCC consultation, we did not specifically collect data on such side effects. This might be a valuable part of follow-up research. Furthermore, we did not correct for possible confounders such as lifestyle factors in our analysis. The development of a model with the hypothesised pathways between LDL-C on target and NCC interventions, including all potential confounders of this relation, could potentially help to more fully investigate the association between NCC titration and LDL-C on target. Such causal mediation analysis may be used to investigate the causal role of titration activities relative to other factors associated with NCC in future trials. ${ }^{25} 26$

\section{CONCLUSION}

In conclusion, among patients hospitalised for ACS, NCC resulted in more intensive medication titration compared with usual care alone. The greater proportion of patients on LDL-C target at 6 and 12 months follow-up is likely explained by the more intensive titration of lipid-lowering medication in NCC patients compared with usual care alone. Merely starting the guideline-recommended dose is insufficient to reach the guideline-recommended LDL-C target level. NCC, combined with guideline-based titration recommendations, can improve ACS patient outcomes and should become part of routine daily practice.

Contributors MS, HTJ, GtR, SMB, WJMSR and RJG participated in the design of secondary analysis. HTJ was responsible for the coordination and acquisition of the trial data. MS, HTJ and MH attributed to the draft versions of the manuscript. MS, HTJ, MH and GtR performed the statistical analysis. All authors contributed to the preparation, critical review and approved the final manuscript.

Competing interests None declared.

Patient consent Detail has been removed from this case description/these case descriptions to ensure anonymity. The editors and reviewers have seen the detailed information available and are satisfied that the information backs up the case the authors are making.

Ethics approval AMC Amsterdam Medical Ethics committee.

Provenance and peer review Not commissioned; externally peer reviewed.

Open Access This is an Open Access article distributed in accordance with the terms of the Creative Commons Attribution (CC BY 4.0) license, which permits others to distribute, remix, adapt and build upon this work, for commercial use, provided the original work is properly cited. See: http://creativecommons.org/licenses/by/4.0/ 
(c) Article author(s) (or their employer(s) unless otherwise stated in the text of the article) 2017. All rights reserved. No commercial use is permitted unless otherwise expressly granted.

\section{REFERENCES}

1. Piepoli MF, Hoes AW, Agewall S, et al. 2016 European Guidelines on cardiovasculardisease prevention in clinical practice. Eur Heart $J$ 2016;2016:2315-81.

2. Kotseva K, De Bacquer D, Jennings C, et al. Time Trends in Lifestyle, Risk Factor Control, and Use of Evidence-Based Medications in Patients With Coronary Heart Disease in Europe: Results From 3 EUROASPIRE Surveys, 1999-2013. Glob Heart 2016;2013.

3. Kotseva K, Wood D, De Backer G, et al. EUROASPIRE III: a survey on the lifestyle, risk factors and use of cardioprotective drug therapies in coronary patients from 22 European countries. Eur $J$ Cardiovasc Prev Rehabil 2009;16:121-37.

4. Kotseva K, Wood D, De Bacquer D, et al. EUROASPIRE IV: A European Society of Cardiology survey on the lifestyle, risk factor and therapeutic management of coronary patients from 24 European countries. Eur J Prev Cardiol 2016;23:636-48.

5. Kotseva K, Wood D, De Backer G, et al. Cardiovascular prevention guidelines in daily practice: a comparison of EUROASPIRE I, II, and III surveys in eight European countries. Lancet 2009;373:929-40.

6. Snaterse M, Dobber J, Jepma P, et al. Effective components of nurse-coordinated care to prevent recurrent coronary events: a systematic review and meta-analysis. Heart 2016;102:50-6.

7. van Halewijn G, Deckers J, Tay HY, et al. Lessons from contemporary trials of cardiovascular prevention and rehabilitation: A systematic review and meta-analysis. Int J Cardiol 2017;232:294-303.

8. Jorstad HT, Chan YK, Scholte op Reimer WJ, et al. Nurses' perspectives on nurse-coordinated prevention programmes in secondary prevention of cardiovascular disease: a pilot survey. Contemp Nurse 2015;51:96-106.

9. Jorstad HT, von Birgelen C, Alings AM, et al. Effect of a nursecoordinated prevention programme on cardiovascular risk after an acute coronary syndrome: main results of the RESPONSE randomised trial. Heart 2013;99:1421-30.

10. Graham I, Atar D, Borch-Johnsen K, et al. European guidelines on cardiovascular disease prevention in clinical practice: full text. fourth Joint Task Force of the european Society of Cardiology and other societies on cardiovascular disease prevention in clinical practice (constituted by representatives of nine societies and by invited experts). Eur J Cardiovasc Prev Rehabil 2007;14 Suppl 2(Supp 2):S1-113.

11. Jørstad HT, Alings AM, Liem AH, et al. RESPONSE study: Randomised Evaluation of Secondary Prevention by Outpatient Nurse SpEcialists: Study design, objectives and expected results. Neth Heart J 2009;17:322-8.

12. Besseling J, Kindt I, Hof M, et al. Severe heterozygous familial hypercholesterolemia and risk for cardiovascular disease: a study of a cohort of 14,000 mutation carriers. Atherosclerosis 2014;233:219-23.
13. NHG Dutch College of General Practitioners. NHG Dutch guideline cardiovascular risk management. Utrecht: NHG Dutch College of General Practitioners, 2009.

14. Penning-van Beest FJ, Termorshuizen F, Goettsch WG, et al. Adherence to evidence-based statin guidelines reduces the risk of hospitalizations for acute myocardial infarction by $40 \%$ : a cohort study. Eur Heart J 2007;28:154-9.

15. Perk J, De Backer G, Gohlke H, et al. European guidelines on cardiovascular disease prevention in clinical practice (version 2012) : the fifth joint task force of the european society of cardiology and other societies on cardiovascular disease prevention in clinical practice (constituted by representatives of nine societies and by invited experts). Int J Behav Med 2012;19:403-88.

16. Shaw RJ, McDuffie JR, Hendrix CC, et al. Effects of nurse-managed protocols in the outpatient management of adults with chronic conditions: a systematic review and meta-analysis. Ann Intern Med 2014;161:113-21.

17. Voogdt-Pruis HR, Van Ree JW, Gorgels AP, et al. Adherence to a guideline on cardiovascular prevention: a comparison between general practitioners and practice nurses. Int J Nurs Stud 2011;48:798-807.

18. Jiang X, Sit JW, Wong TK. A nurse-led cardiac rehabilitation programme improves health behaviours and cardiac physiological risk parameters: evidence from Chengdu, China. J Clin Nurs 2007;16:1886-97.

19. Laurant $\mathrm{M}$, Harmsen $\mathrm{M}$, Wollersheim $\mathrm{H}$, et al. The impact of nonphysician clinicians: do they improve the quality and costeffectiveness of health care services? Med Care Res Rev 2009;66:36S-89.

20. Jackevicius CA, Li P, Tu JV, Prevalence TJV. Prevalence, predictors, and outcomes of primary nonadherence after acute myocardial infarction. Circulation 2008;117:1028-36.

21. Baigent C, Blackwell L, Emberson J, et al. Efficacy and safety of more intensive lowering of LDL cholesterol: a meta-analysis of data from 170,000 participants in 26 randomised trials. Lancet 2010;376:1670-81

22. Boekholdt SM, Hovingh GK, Mora S, et al. Very low levels of atherogenic lipoproteins and the risk for cardiovascular events: a meta-analysis of statin trials. J Am Coll Cardiol 2014;64:485-94.

23. Baigent $C$, Keech A, Kearney PM, et al. Efficacy and safety of cholesterol-lowering treatment: prospective meta-analysis of data from 90,056 participants in 14 randomised trials of statins. Lancet 2005;366:1267-78.

24. Jørstad HT, Minneboo M, Helmes HJ, et al. Effects of a nursecoordinated prevention programme on health-related quality of life and depression in patients with an acute coronary syndrome: results from the RESPONSE randomised controlled trial. BMC Cardiovasc Disord 2016;16:144-016.

25. Imai K, Keele L, Tingley D. A general approach to causal mediation analysis. Psychol Methods 2010;15:309-34.

26. Bind MA, Vanderweele TJ, Coull BA, et al. Causal mediation analysis for longitudinal data with exogenous exposure. Biostatistics 2016;17:122-34. 